\title{
Orbital structure of triaxial galaxies
}

\author{
Glenn van de Ven, Ellen Verolme, Michele Cappellari, Tim de Zeeuw \\ Sterrewacht Leiden, Postbus 9513, 2300 RA Leiden, The Netherlands
}

\begin{abstract}
We have developed a method to construct realistic triaxial dynamical models for elliptical galaxies, allowing us to derive best-fitting parameters, such as the mass-to-light ratio and the black hole mass, and to study the orbital structure. We use triaxial theoretical Abel models to investigate the robustness of the method.
\end{abstract}

\section{Triaxial dynamical models}

Many elliptical galaxies show significant signatures of triaxiality (e.g. de Zeeuw et al. 2002). Therefore, we have extended Schwarzschild's orbit superposition method to construct realistic triaxial dynamical models, which fit the observed surface brightness, as well as (two-dimensional) kinematical measurements of elliptical galaxies (Verolme et al. 2003). This fully numerical method is, however, too computationally expensive to do a full search over the model parameters, such as mass-to-light ratio, black hole mass, viewing direction and intrinsic shape. Approximating the potential by one of Stäckel form, we can construct velocity and velocity dispersion fields using the analytical solution of the continuity equation and the three Jeans equations (Statler 1994, van de Ven et al. 2003), and compare them with observations to constrain the large parameter range. Within this reduced parameter space, we can then apply the extended Schwarzschild method using the true potential, to find the true best-fitting triaxial model. Schwarzschild's method not only provides the best-fitting parameters, but also results in an orbital weight distribution, which after appropriate smoothing allows us to study the orbital structure of the observed galaxy.

\section{Triaxial Abel models}

To investigate the robustness of the derived internal structure, we are applying our method to theoretical models with known distribution function (DF). We use Abel models (Dejonghe \& Laurent 1991; Mathieu \& Dejonghe 1999), for which the potential is assumed to be of Stäckel form and the DF to be a function of a single parameter $F\left(E, I_{2}, I_{3}\right)=F(S)$, with $S=E+w I_{2}+u I_{3}$ a linear combination of the explicitly known integrals of motion. The density and higher velocity moments can be calculated efficiently. Besides this analytical simplicity, the Abel models have, with the choice of a three-integral DF, enough freedom to incorporate many of the observed triaxial features (Figure 1). 


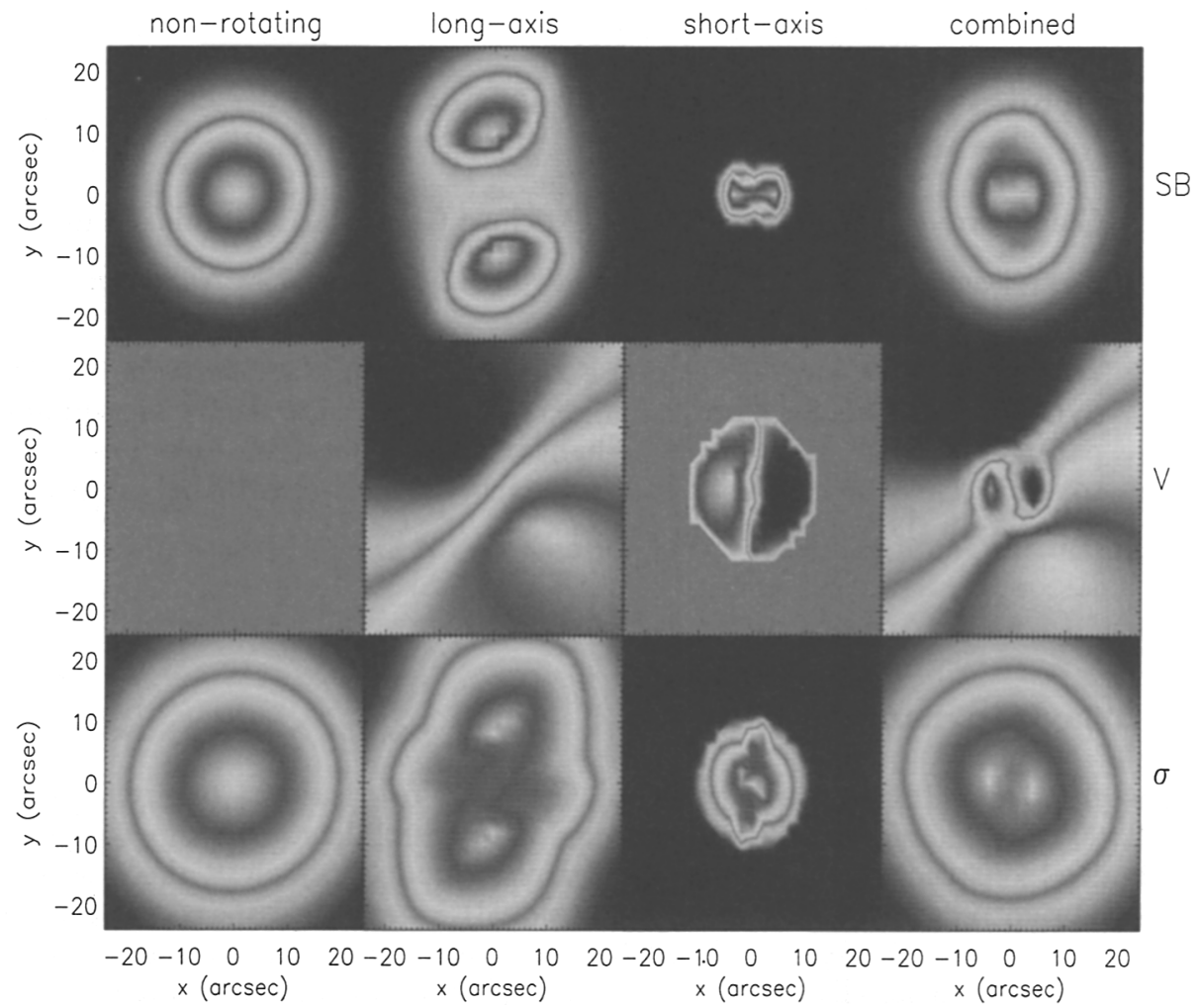

Figure 1. Kinematics of a triaxial Abel model. From top to bottom: the surface brightness (SB), the mean velocity $(V)$ and velocity dispersion $(\sigma)$. From left to right: non-rotating, (outer and inner) long-axis and short-axis rotating components, and a (luminosity weighted) combination. The non-rotating components have zero mean velocity. The short-axis component is limited due to the finite extent of Abel models. The kinematically decoupled core in the combined model is a typical signature of triaxiality observed in e.g. NGC 4365 (Davies et al. 2001).

\section{References}

Davies R. L. et al. 2001, ApJ, 548, L33

Dejonghe, H., \& Laurent, D. 1991, MNRAS, 252, 606

de Zeeuw, P. T. et al. 2002, MNRAS, 283, 149

Mathieu, A., \& Dejonghe, H. 1999, MNRAS, 303, 455

Statler, T. S. 1994, ApJ, 425, 458

van de Ven, G., Hunter, C., Verolme, E. K., \& de Zeeuw, P. T. 2003, MNRAS, 342,1056

Verolme, E. K., Cappellari, M., Emsellem, E., van de Ven, G., \& de Zeeuw, P. T. 2003, MNRAS, submitted 